\title{
Stability of Cortical Thinning in Persons at Increased Familial Risk for Major Depressive Disorder Across 8 Years
}

\author{
Xuejun Hao, Ardesheer Talati, Stewart A. Shankman, Jun Liu, Jürgen Kayser, Craig E. Tenke, \\ Virginia Warner, David Semanek, Priya J. Wickramaratne, Myrna M. Weissman, and \\ Jonathan Posner
}

\begin{abstract}
BACKGROUND: A biological marker of vulnerability should precede onset of illness and be independent of disease course. We previously reported that cortical thinning may serve as a potential biomarker for risk for familial depression. We now test stability of cortical thinning across 8 years, and whether thinning mediates associations between familial risk and depressive traits.

METHOD: Participants were from a three-generation family study of depression, where second- and third-generation offspring were characterized as being at high or low risk for depression based on the presence/absence of major depressive disorder in the first generation. The analysis includes 82 offspring with anatomical magnetic resonance imaging scans across two assessment waves conducted 7.8 years apart (SD = 1.3 years; range, 5.2-10.9 years). RESULTS: High-risk offspring had thinner bilateral superior and middle frontal gyri and left inferior parietal lobule at both time points. High intrasubject correlation $(.60<r<.91)$ and intraclass correlation $(0.72-0.78)$ of thickness measures across time points was detected within the above regions; rank order by effect size and region was also preserved across time. The thinning was stable despite changes in scanning platform (Siemens Sonata vs. GE Signa), field strength (1.5T vs. 3T), and participant age and clinical course. Thinning at the first time point predicted anger and hostility at the second time point and mediated the relationship between familial risk and these traits.

CONCLUSIONS: The study provides evidence for cortical thinning as a stable biomarker for familial vulnerability for depressive illness, which supports the ability to detect persistent and clinically relevant anatomical findings regardless of magnetic resonance imaging platform.
\end{abstract}

Keywords: Biomarker, Depression, FreeSurfer, High-risk, MRI, Stability

http://dx.doi.org/10.1016/j.bpsc.2017.04.009

A central goal in psychiatric neuroscience is to identify abnormalities in brain structure and function that predispose to mental illnesses. To represent true markers of risk, such anomalies [also referred to as "endophenotypes" $(1,2)$ ] should precede onset of the disorder itself, be distinct from changes in the brain that occur as a result of the illness, and be stable over time. This last criterion is cumbersome to demonstrate, as it requires that individuals be scanned at more than one time point while keeping methodological variation to a minimum. Most longitudinal studies have focused on tracking changes in the brain as a function of illness; few have explicitly tested the stability of brain phenotypes.

We previously reported on a potential biomarker for depression vulnerability in a three-generation family study of major depressive disorder (MDD) (3). We found that secondand third-generation offspring at high compared to low familial risk for depression (where we defined familial risk based on the presence or absence of MDD in the first-generation probands) had thinner cortices, particularly in the lateral surface of the right hemisphere. The thinning was present even in offspring who were at risk but who had never had an episode of MDD, suggesting that it was unlikely to be a consequence of the illness. We hypothesized that cortical thinning may represent an endophenotype for the familial form of MDD.

What we could not test in our previous report was whether the thinning represented a stable trait, as participants had only been imaged at one time point. We have since rescanned the population, on average 7.8 (range, 5.2-10.9) years later, which allowed us to examine the extent to which differences in cortical morphology between the high- and low-risk groups were conserved over time. In testing this, we addressed three goals. First, we tested if cortical thinning was still present 8 years later. If so, this stability would increase confidence that cortical thinning reflects a stable biomarker rather than a transient phenotype or a clinical state. Second, we tested whether the cortical thinning was robust to methodological 
variation, such as the magnetic resonance imaging (MRI) scanning site, platform, and magnetic field strength. For the development of biomarkers, it is critical to test potential effects of methodological advances and to disentangle heterogeneity attributable to biologically relevant processes from that caused by methodological sources. Third, our original findings were reported using proprietary anatomical MRI methods that are not widely available. To foster greater reproducibility, we now use FreeSurfer $(4,5)$, a freely available, open-source software that is among the most frequently used techniques for examining cortical morphology. FreeSurfer is also optimized for longitudinal analyses because its algorithms allow for greater control over segmentation differences across scans than do other software packages (6).

The primary goal of this study was to test whether differences in cortical thickness related to familial risk for depression are stable over an 8-year period, where stability was defined by test-retest consistency of anatomy (i.e., Do brain regions showing significant differences in cortical thickness at the first time point also do so at the second?) and rank order (i.e., Do participants with the greatest degree of thinning at the first time point continue to do so at the second?). Finally, to better understand the clinical implications of cortical thinning, we explored whether cortical thinning identified at the first scan predicted clinical phenotypes relevant to depression approximately 8 years later.

\section{METHODS AND MATERIALS}

\section{Participants}

The sample has been detailed in several publications (2,7-9). Briefly, the study began in 1982 with the simultaneous recruitment of two groups of probands (generation 1 [G1]). Depressed probands were selected from outpatient psychiatric clinics for the treatment of mood disorders in the New Haven, Connecticut, area and were required to have moderate to severe MDD. Nondepressed probands were selected concurrently from the same community and were required to have no lifetime history of psychiatric illness, based on several interviews. All probands were of European ancestry. Their biological children (G2) and subsequently grandchildren (G3) were followed prospectively over time. The offspring of the depressed probands formed the "high-risk" group, and those of the nondepressed probands formed the "low-risk" group $(8,9)$.

\section{Assessments}

Diagnostic interviews were conducted using the adult (10) or child (6-17 years) (11) version of the semistructured Schedule for Affective Disorders and Schizophrenia-Lifetime interview by doctoral and masters level mental health professionals [reliability was high, as documented elsewhere $(8,9)]$. The first interview assessed the lifespan to that point; follow-up interviews assessed catch-up periods; diagnoses are therefore cumulative until the latest interview. Each family member was interviewed independently and blinded to the clinical status of other family members. Final diagnoses were made by one or more experienced clinicians, using the best-estimate procedure (12). Current depressive symptoms at the time of each scan were measured using the Hamilton Depression Rating Scale (13) or Children's Depression Rating Scale, Revised (14) (for adults and children, respectively); anxiety symptoms were measured with the Hamilton Anxiety Rating Scale (15) and revised Children's Manifest Anxiety Scale (16), respectively. Child and adult scores were first each converted to $z$ scores, which were then combined to create a single measure. In addition to state measures, we also collected trait measures of impulsivity [Barratt Impulsiveness Scale version 11 (17)], and anger and hostility [Buss-Perry Aggression Questionnaire $(18,19)]$. These measures were assessed because our primary hypothesis suggests that cortical thinning represents a stable biomarker of risk for depression, and thus its clinical correlates should likewise be stable, trait markers of depression risk.

\section{Analytic Sample}

We obtained MRI scans from 158 G2 and G3 offspring between 7 and 55 years of age at wave 5 (W5) and from 114 offspring between 11 and 68 years of age at wave 6 (W6). MRI scans from 8 individuals at W5 and 1 individual at W6 were excluded because of severe head motion. Of 150 usable W5 scans and 113 usable W6 scans, there were 82 common individuals (43 from high-risk families and 39 from low-risk families). The interval between W5 and W6 scans for the same individual varied from 5.2 to 10.9 years, with a mean of 7.8 and SD of 1.3 years. Interscan intervals were similar for G2 (7.6 \pm 1.4 years) and G3 (8.1 \pm 1.2 years) individuals.

Analyses on clinical correlates of cortical thinning were based on the 110 (of 150) individuals with a usable MRI scan at W5 who also had clinical measures at W6. These 110 participants did not differ on measures of age, sex, or risk status from the primary sample of $n=82$ with usable brain scans at both waves (not shown).

\section{MRI Scanning}

W5 MRI scans were obtained using a Siemens Sonata 1.5T scanner (Siemens AG, Munich, Germany) using a threedimensional magnetization prepared rapid acquisition gradient-echo sequence (repetition time $=24 \mathrm{~ms}$, echo time $=$ $2.96 \mathrm{~ms}, 45^{\circ}$ flip angle, field of view $=30 \times 30 \mathrm{~cm}$, phase field of view $=100 \%, 2$ excitations, $1.2-\mathrm{mm}$ slice thickness, $256 \times$ 192 matrix size, 128 slices, $1.17 \times 1.17 \times 1.2 \mathrm{~mm}$ voxel resolution). W6 MRI scans were obtained using a GE Signa 3T whole-body scanner (GE Medical Systems, Milwaukee, WI) equipped with an 8-channel, phased array head coil using three-dimensional fast spoiled gradient recall sequence (repetition time $=4.7 \mathrm{~ms}$, echo time $=1.3 \mathrm{~ms}, 110^{\circ}$ flip angle, bandwidth $=41.67 \mathrm{MHz}$, field of view $=25 \times 25 \mathrm{~cm}$, array spatial sensitivity encoding technique factor $=2,1-\mathrm{mm}$ slice thickness, $256 \times 256$ matrix size, 128 slices, $0.98 \times 0.98 \times$ $1.0 \mathrm{~mm}$ voxel resolution, $1 \mathrm{NEX}$ images $\times 2$ ).

\section{Processing Pipeline}

Methods for estimating cortical thickness can be broadly categorized as surface- or voxel-based. We used FreeSurfer $(4,5)$, a surface-based approach to estimate cortical thickness at each point on the pial surface. The use of explicit surface models enables subvoxel accuracy, high sensitivity, and 
robustness to different field strengths, scanner upgrade, and scanner manufacturer (20).

The processing pipeline was as follows. First, large-scale variations in image intensity are corrected using bias field estimation and bias removal. Second, extracerebral tissues are removed using an automated tool. Third, an initial segmentation separates gray matter, white matter, and cerebrospinal fluid. The white matter/gray matter boundary is referred to as the white matter boundary, and the gray matter/cerebrospinal fluid boundary as the pial boundary. With this initial segmentation, FreeSurfer constructs a surface of triangulated mesh based on the white matter boundary, which is then deformed to find the opposing pial boundary, correcting topological defects using smoothness and self-intersection constraints. With the cortex closed at the brain stem, the resulting surface was topologically equivalent to a sphere. The closest distance from the white surface to the pial surface at each surface's vertex is then defined as the cortical thickness.

Processed data were visually inspected and manually edited by two independent technicians (one per wave) who were well-trained in neuroanatomy to correct for errors from the automated pipeline, such as skull strip errors, segmentation errors, and intensity normalization failure. Data processing was blinded to the subject's risk status. Thus, interrater differences are unlikely to generate the observed group differences (thinning in high-risk offspring) at either wave or the corresponding of group differences across waves.

\section{Statistical Analyses}

We examined the relationship between cortical thickness and risk status (high or low) using a general linear model with each vertex across the pial boundary as the dependent variable, risk status as the independent variable, and age at current scan and sex as covariates. $P$ values were color coded and plotted for each vertex at the pial boundary and results controlled for the false discovery rate (21).

We explored clinical correlates of cortical thinning using path analysis. Specifically, we tested whether cortical thinning mediates the relationship between familial risk and behavioral traits associated with depression. Following established methods for path analysis (22), we conducted a series of linear regression analyses. We first tested whether the mediating variable, cortical thinning, was associated with risk status. We then tested whether cortical thinning correlated with depressive symptoms and/or traits, while controlling for risk status. Statistical significance was then determined based a 95\% confidence interval derived from the Sobel test and confirmed by bias-corrected bootstrapping with 2000 samples (not shown). Age at first scan, age difference between the two scans, and sex, were included as covariates in all of the path linear regression models and in the Sobel test and bootstrapping.

\section{RESULTS}

Participants at high compared to low familial risk for depression were approximately 8 years older at each scan (range, 5-11 years) (Table 1). Within each generation of offspring, age did not vary by risk status; however, a greater proportion of G2 offspring, as compared with G3 offspring,
Table 1. Demographic and Clinical Characteristics of 82 Participants With Wave 5 and Wave 6 Scans

\begin{tabular}{lcccc}
\hline & $\begin{array}{c}\text { High-Risk } \\
(n=43)\end{array}$ & $\begin{array}{c}\text { Low-Risk } \\
(n=39)\end{array}$ & $\begin{array}{c}\text { Test } \\
\text { Statistic }\end{array}$ & $\begin{array}{c}p \\
\text { Value }\end{array}$ \\
\hline Age, Years & & & & \\
\hline W5 & $31.7 \pm 12.8$ & $23.1 \pm 12.8$ & $t=3.1$ & .003 \\
\hline W6 & $39.4 \pm 12.7$ & $31.2 \pm 12.8$ & $t=2.9$ & .005
\end{tabular}

Age, By Generation, Years

\begin{tabular}{|c|c|c|c|c|}
\hline \multicolumn{5}{|l|}{ G2 } \\
\hline W5 & $38.7 \pm 8.1$ & $39.7 \pm 5.6$ & $t=0.5$ & .63 \\
\hline W6 & $46.2 \pm 8.1$ & $47.8 \pm 5.9$ & $t=0.7$ & .48 \\
\hline \multicolumn{5}{|l|}{ G3 } \\
\hline W5 & $15.8 \pm 5.0$ & $14.7 \pm 3.9$ & $t=0.7$ & .50 \\
\hline W6 & $23.8 \pm 4.9$ & $23.0 \pm 4.3$ & $t=0.5$ & .62 \\
\hline \multicolumn{5}{|l|}{ Generation } \\
\hline G2 & $30(69.8)$ & $13(33.3)$ & $\chi^{2}=10.9$ & .001 \\
\hline G3 & $13(30.2)$ & $26(66.7)$ & & \\
\hline \multicolumn{5}{|l|}{ Sex } \\
\hline Male & $20(46.5)$ & 17 (43.6) & $\chi^{2}=0.07$ & .79 \\
\hline Female & $23(53.5)$ & $22(56.4)$ & & \\
\hline \multicolumn{5}{|l|}{ Depressive Symptoms ${ }^{a}$} \\
\hline \multicolumn{5}{|l|}{ Adult } \\
\hline W5 & $4 \pm 4.9$ & $3.2 \pm 4.2$ & $t=0.6$ & .55 \\
\hline W6 & $3.6 \pm 5.1$ & $1.4 \pm 4.5$ & $t=2.0$ & .05 \\
\hline \multicolumn{5}{|l|}{ Child } \\
\hline W5 & $20.2 \pm 9.0$ & $21.0 \pm 7.1$ & $t=0.2$ & .82 \\
\hline W6 & $21.3 \pm 4.5$ & $18.0 \pm 16.7$ & $t=0.3$ & .77 \\
\hline \multicolumn{5}{|l|}{ Anxiety Symptoms ${ }^{b}$} \\
\hline \multicolumn{5}{|l|}{ Adult } \\
\hline W5 & $3.7 \pm 4.1$ & $2.5 \pm 3.5$ & $t=1.1$ & .28 \\
\hline W6 & $2.6 \pm 3.4$ & $1.0 \pm 3.0$ & $t=2.2$ & .04 \\
\hline \multicolumn{5}{|l|}{ Child } \\
\hline W5 & $8.7 \pm 8.3$ & $5.8 \pm 6.7$ & $t=0.9$ & .37 \\
\hline W6 & $2.3 \pm 3.2$ & $3.0 \pm 5.2$ & $t=0.3$ & .86 \\
\hline $\begin{array}{l}\text { Current/Lifetime } \\
\text { Depressive Disorder }\end{array}$ & $24(55.8)$ & $12(30.8)$ & $\chi^{2}=5.2$ & .02 \\
\hline $\begin{array}{l}\text { Current/Lifetime Anxiety } \\
\text { Disorder }\end{array}$ & $28(65.1)$ & $19(48.7)$ & $\chi^{2}=2.2$ & .13 \\
\hline
\end{tabular}

Values are expressed as mean \pm SD or $n(\%)$.

Symptom scores were Z-transformed for adults and children independently before they were included as covariates in the statistical models.

G2, generation 2; G3, generation 3; W5, wave 5; W6, wave 6 .

${ }^{a}$ Depressive symptoms were determined using the Hamilton Depression Rating Scale and the Children's Depression Inventory for adults and children, respectively.

${ }^{b}$ Anxiety symptoms were determined using the Hamilton Anxiety Rating Scale and the Revised Children's Manifest Anxiety Scale for adults and children, respectively.

were from high-risk families. Individuals from high-risk families had higher lifetime rates of depressive disorders but not anxiety disorders.

Statistically significant thinning was found in the high-risk group compared with the low-risk group in bilateral superior frontal and caudal middle frontal cortices and the left inferior parietal lobule (Figure 1A, thinning in blue). Thinning patterns were consistent across the first (W5, left) and second (W6, 


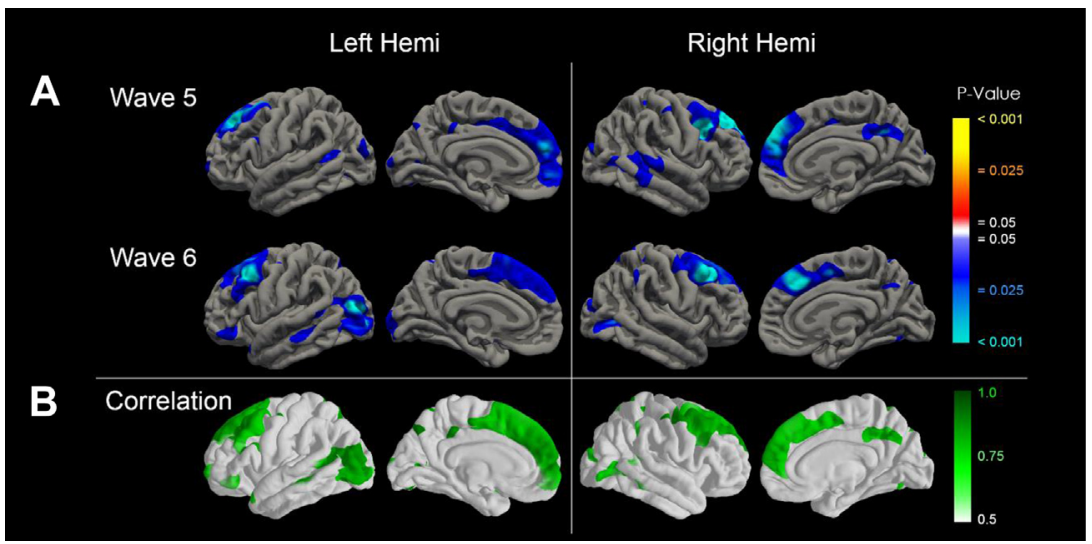

Figure 1. Maps of group differences in cortica thickness of 82 common subjects (43 high-risk, 39 low-risk) at waves 5 and 6. (A) At each point on the cerebral surface, the statistical significance (probability values) of differences in cortical thickness across groups (high- vs. low-risk) in participants from generations 2 and 3 are color coded. Warm colors (yellow, orange, and red) represent significantly thicker cortices in the high-risk group; cooler colors (blue and cyan) represent thinner cortices in that group. The color bar indicates the color coding of $p$ values for testing of statistical significance at each point on the surface of the brain. The statistical models controlled for the age and sex of all participants. (B) Plot of Pearson correlation coefficients of cortical thickness between waves 5 and 6 in conjunct thinning regions. Hemi, hemisphere.

right) scans. As shown in Table 2, not only were the same cortical regions identified at W5 and W6, but the rank order of findings - that is, the relative effect sizes of the group thickness differences ( $\beta$ s)-were preserved.

Within regions in which significant group effects were detected, we plotted within-participant correlations of cortical thickness from W5 to W6. As shown in Figure 1B, high intrasubject correlation $(r=.60-.91)$ and intraclass correlation (absolute agreement $=.72-.78$ ) from W5 to W6 further supports the stability of cortical thinning in high-risk individuals across waves.

\section{Cortical Thinning Mediates the Relationships Between Familial Risk and Anger and Hostility}

We explored associations between cortical thinning at W5 and depression-related traits measured at W6 (Figure 2). Familial risk status predicted both anger (left, $\beta=4.87, p=.017$ ) and hostility (right, $\beta=3.50, p=.021$ ) but not impulsivity or other facets of aggression (physical or verbal aggression). Within each hemisphere of the superior frontal gyrus, thinner cortices predicted greater anger and hostility, and the thinning mediated the relationship between familial risk and trait measures (mediating paths shown in red). Cortical thinning was not associated with current depressive symptom severity, lifetime history of depression, or impulsivity.

Table 2. Cortical Regions of Interest With Significant Thinning at Either Wave of Assessment

\begin{tabular}{|c|c|c|c|c|}
\hline \multirow[b]{2}{*}{ Cortical Region } & \multicolumn{2}{|c|}{ Wave 5} & \multicolumn{2}{|c|}{ Wave 6} \\
\hline & $\begin{array}{l}\text { Size } \\
\left(\mathrm{mm}^{2}\right)\end{array}$ & $\begin{array}{c}\beta \text { Mean } \\
(\mathrm{mm})\end{array}$ & $\begin{array}{l}\text { Size } \\
\left(\mathrm{mm}^{2}\right)\end{array}$ & $\begin{array}{c}\beta \text { Mean } \\
(\mathrm{mm})\end{array}$ \\
\hline Right Superior Frontal & 2983.5 & -0.158 & 2985.1 & -0.112 \\
\hline Left Superior Frontal & 3835.0 & -0.143 & 4023.9 & -0.105 \\
\hline $\begin{array}{l}\text { Left Caudal Middle } \\
\text { Frontal }\end{array}$ & 1200.4 & -0.141 & 1020.2 & -0.102 \\
\hline $\begin{array}{l}\text { Right Caudal Middle } \\
\text { Frontal }\end{array}$ & 1411.2 & -0.137 & 1291.0 & -0.098 \\
\hline Left Inferior Parietal & 850.4 & -0.093 & 772.2 & -0.096 \\
\hline
\end{tabular}

The size of thinning regions and their corresponding average $\beta$ values are reported here for statistically significant regions.

\section{Supplementary Analyses}

Because there were a number of co-occurring changes from W5 to W6 (e.g., MRI scanner and field strength, development stage and age, diagnostic status, substance use, or medication), we conducted sensitivity analyses to test whether any of these changes could confound the main results. The findings, shown in Supplemental Figures S1-S5, indicate that the group differences in cortical thickness remained stable and could not be attributed to any of the above factors.

\section{DISCUSSION}

We show that familial risk for depression is related to cortical thinning in bilateral superior and caudal middle frontal gyri and the left inferior parietal lobule. The thinning is stable across approximately 8 years, consistent with the hypothesis that cortical thinning reflects a stable trait. The stability was robust to methodological variation, including changes in MRI scanner (Siemens and GE) or field strength (1.5T and 3T), and to individual variation across participants (age and clinical trajectories). Finally, thinning in bilateral frontal cortices mediated the relationship between familial risk and trait measures of anger and hostility, suggesting a potential pathway by which familial risk for depression may contribute to the biological (cortical thinning) and clinical (anger/hostility) presentation of offspring. This is to our knowledge the largest and longest study of its kind, demonstrating the stability of a biomarker of risk for depression.

\section{Stability Across 8 Years}

The implications of the consistency of findings across 8 years are multifold. From a clinical perspective, the stability, coupled with our previous (3) and current (Supplemental Figure S4) observations that the thinning is present even in offspring who are at risk but who never develop depression, further validates cortical thinning as a risk marker. At the same time, the high reliability of findings across different scanning platforms and field strengths suggest that detection is resistant to methodological variation. The consistency of findings across $1.5 \mathrm{~T}$ and 3T scanning platforms is particularly noteworthy, given the lower resolution with the former (the voxel size and in-plane area were $71 \%$ and $43 \%$ larger, respectively). Many early 


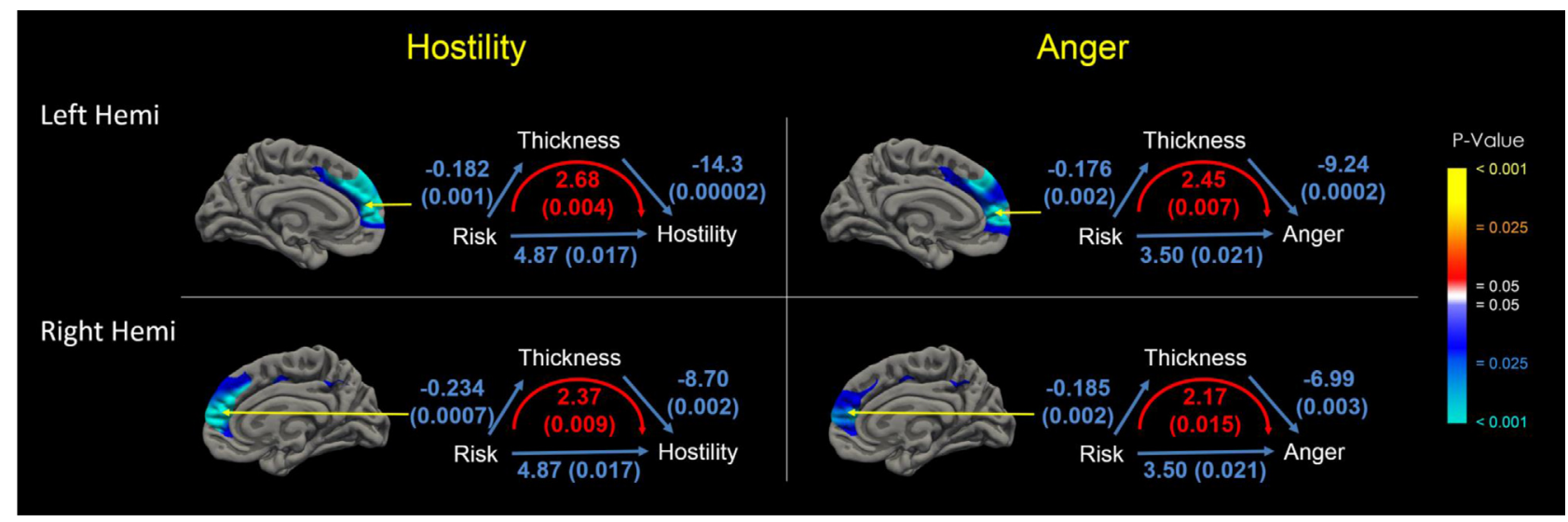

Figure 2. Correlation and mediation of cortical thickness with measures of aggression hostility. Brain images show correlation of cortical thickness at the first scan (i.e., wave 5) with measures of hostility (left) and anger (right). Regions shown in warm colors (yellow to red) represent those where thicker cortex was associated with higher hostility/anger scores; there were no regions meeting these criteria. Regions shown in cool colors (blue and cyan) represent those where thinner cortex was associated with higher hostility/anger. The color bar on the right indicates the color coding of $p$ values for testing of statistical significance at each point on the surface of the brain. The arrow diagrams illustrate the pathways tested in the mediation models, with blue pathways indicating the three direct paths and the red pathway indicating the indirect path. Each path lists the $\beta$ values (adjusted for age at first scan, age difference between scans, and sex), with corresponding $p$ values in parentheses. The red path indicates the indirect path-the portion of the association between familial risk and anger/ hostility outcomes that is mediated (explained) by the cortical thinning in the respective region. Hemi, hemisphere.

MRI studies on depression were performed on 1.5T scanners (23-26). In recent years, 3T scanners have become the norm, and because the technology for neuroimaging is constantly improving, methods and field strengths will continue to evolve. Showing stability across different platforms will be particularly important for meta-analyses and systematic reviews, where consistency of data quality across space and time is essential, yet often assumed rather than empirically investigated.

Establishing reliability has been problematic in psychiatric research. This, in part, may be because of the substantial diagnostic heterogeneity within psychiatric nosology (27), and in MRI studies is exacerbated by the risks for false positive findings that in some cases may exceed $50 \%(28,29)$. These hurdles against identifying reliable biomarkers underscore the importance of within-study, test-retest reliability. Our use of open-source software will permit external groups to replicate and extend findings. Although caution is always necessary when attempting to integrate data acquired through different platforms, our findings suggest that data acquired on 1.5T scanners may be at least qualitatively comparable with newer data (see Limitations).

The thinning observed here was only partially consistent with the thinning we previously reported using different voxelbased methods (3). Although we found that offspring at high familial risk had significant thinning of the cortical mantle in both reports, the thinning that we previously reported was more globally distributed through parietal, temporal, and frontal cortices and predominantly in the lateral right and medial left hemisphere-an asymmetry we do not find here. Differences could be caused by the only partially overlapping samples or because of the different approaches implemented. Surface-based estimations (as implemented here with FreeSurfer) may be preferable for longitudinal analyses because of deformable model processing, which optimizes correction against segmentation differences across scans (6). Conversely, voxel-based methods, which measure thickness directly from initial segmentation, are more prone to segmentation errors. Our use of open-source software will now allow other investigators to compare patterns of cortical thinning in independent samples using parallel methods.

\section{Cortical Thinning in Depressive Disorders}

A number of previous studies have reported reductions in cortical thickness in frontal and temporal lobes in depressive disorders (30-33) [reviews (27,34)]. The thinning has been further associated with atypical functional connectivity (33) and executive functioning $(32,35)$, and with depression phenotypes in other disorders [e.g., diabetes (35) and Alzheimer's dementia (36)]. However, most studies to date have only compared individuals with and without depression, precluding disentanglement of brain differences that predispose to the disorder from those that may arise as a consequence of the illness. By identifying subjects based on their risk for rather than the presence of the outcome of interest, high-risk designs allow us to target biological processes that emerge before disease onset (37). Few imaging studies have applied this approach to depression. A study by Papmeyer et al. followed early adult offspring (mean age $=21$ years) at high and low familial risk for depression, but only across a 2-year period (31). Offspring at high risk for depression had thinner right temporal cortex, regardless of whether they developed the disorder themselves. Whereas high-risk offspring who did not develop depression showed thinning across the 2 years in inferior frontal cortex, those who did develop depression showed corresponding thickening, which the authors suggest may be a function of insufficient synaptic pruning.

\section{Cortical Thinning Mediates Associations Between Familial Risk and Depression Phenotypes}

Frontal cortical thinning mediated the association between familial risk for depression and two adulthood traits associated 
with depression, hostility, and anger. As illustrated in Figure 2, frontal thinning partially explained the relationship between familial risk and anger and hostility. Interestingly, thinning did not explain associations between familial risk and offspring depression. This could be because depressive symptoms present episodically and thus may be more likely to associate with similarly episodic biological measures. Expression also depends on the environment, and many offspring who may be at high familial risk and have the cortical vulnerability still never go on to develop symptoms.

Though not core clinical symptoms of depression, anger and hostility are reported in about a third of depressed patients $(15,38)$, and are associated with a poorer course of the disorder, including poorer response to pharmacological treatment and higher rates of suicide attempts, accidents, and cardiovascular disease $(39,40)$. Family history may thus lead to some depressions by increasing proneness to aggression traits via dysregulation of circuits involving regions showing frontal thinning. This hypothesis is consistent with studies showing higher levels of aggression in adolescents with thinner middle frontal cortices or disruptions in frontolimbic circuitry (41). Future research can test this hypothesis more directly by following teenage offspring who have both frontal thinning and elevated aggression traits, and test whether the aggression mediates an association between the thinning and later onset of clinical symptoms.

\section{Limitations}

Our sample is based on families who are at high or low risk for MDD, neither of which are completely reflective of the general U.S. population. The sample was also of European ancestry, so findings may not generalize to other groups. Although we show that cortical thinning predicts measures of aggression approximately 8 years later, temporal causality should not be inferred, because the onset for development of the cortical thinning versus behavioral traits is not known. The effect size was also moderate-to-large; for subtler regional differences or more sensitive imaging modalities (e.g., functional MRI), the signal-to-noise ratio may be insufficient to document correspondence across scans. On the other hand, the method variation across scans could have inflated type II errors, leaving us with a conservative estimate of stability. Associations between cortical thickness and impulsivity could be accounted for by third variables (e.g., trauma) that could alter both thickness and trait measures. Finally, the study focused on biomarkers in offspring at high familial risk for depression. Thus, it is unclear whether similar markers would be found for other types of depression that are not family based. Similarly, because the study did not include offspring of probands with other disorders, we cannot empirically test whether the markers are specific to depression versus reflective of broader psychiatric risk.

In conclusion, identifying biomarkers of vulnerability is a critical step in treatment development. A true vulnerability marker must represent risk for illness; simply serving as a onetime correlate of illness is insufficient (1). Using a high-risk family design, we have identified a stable biomarker for depression risk (bilateral frontal thinning) that meets the above criteria. The marker first needs to be reproduced and validated independently. Subsequent research can then address predictive validity by following individuals with frontal thinning over time and examining the long-term clinical trajectories. At the same time, functional imaging approaches can be applied to examine brain-wide circuitry implications of the thinning.

\section{ACKNOWLEDGMENTS AND DISCLOSURES}

This work was supported by National Institute of Mental Health Grant Nos. R01MH036197 (to MMW) and 1P50MH090966 (principal investigator, Jay Gingrich), the Sackler Institute for Developmental Psychobiology, and the Templeton Foundation.

In the last 3 years, MMW has received royalties from Oxford University Press, Perseus Books Group, American Psychiatric Association Publishing, and Multi-Health Systems. JP has received funding from Shire Pharmaceuticals. None of these present any conflict with the present work. All other authors report no biomedical financial interests or potential conflicts of interest.

\section{ARTICLE INFORMATION}

From the Department of Psychiatry (XH, AT, JL, JK, CET, VW, PJW, MMW), Columbia University Medical Center, Divisions of Epidemiology $(X H, A T, J L$, JK, CET, VW, PJW, MMW) and Child Psychiatry (DS, JP), New York State Psychiatric Institute, and the Departments of Epidemiology (MMW) and Biostatistics (PJW), Mailman School of Public Health, Columbia University, New York, New York; and the Department of Psychology and Psychiatry (SAS), University of Illinois at Chicago, Chicago, Illinois.

$\mathrm{XH}$ and $\mathrm{AT}$ contributed equally to this work and are listed in alphabetical order.

Address correspondence to Ardesheer Talati, Ph.D., Department of Psychiatry, Columbia University and New York State Psychiatric Institute, 1051 Riverside Drive, Unit 24, New York, NY 10032; E-mail: talatia@nyspi. columbia.edu.

Received Feb 14, 2017; revised Apr 12, 2017; accepted Apr 24, 2017.

Supplementary material cited in this article is available online at http://dx doi.org/10.1016/j.bpsc.2017.04.009.

\section{REFERENCES}

1. Gottesman II, Gould TD (2003): The endophenotype concept in psychiatry: Etymology and strategic intentions. Am J Psychiatry 160: 636-645.

2. Gould TD, Gottesman II. (2006): Psychiatric endophenotypes and the development of valid animal models. Genes Brain Behav 5:113-119.

3. Peterson BS, Warner V, Bansal R, Zhu H, Hao X, Liu J, et al. (2009): Cortical thinning in persons at increased familial risk for major depression. Proc Natl Acad Sci U S A 106:6273-6278.

4. Dale AM, Fischl B, Sereno MI (1999): Cortical surface-based analysis I. Segmentation and surface reconstruction. Neuroimage 9:179-194.

5. Fischl B, Sereno MI, Dale AM (1999): Cortical surface-based analysis II: Inflation, flattening, and a surface-based coordinate system. Neuroimage 9:195-207.

6. Fisher LB, Fava M, Doros GD, Alpert JE, Henry M, Huz I, et al. (2015): The role of anger/hostility in treatment-resistant depression: A secondary analysis from the ADAPT-A study. J Nerv Ment Dis 203:762-768.

7. Mills KL, Tamnes CK (2014): Methods and considerations for longitudinal structural brain imaging analysis across development. Dev Cogn Neurosci 9:172-190.

8. Weissman MM, Wickramaratne P, Nomura Y, Warner V, Pilowsky D, Verdeli H (2006): Offspring of depressed parents: 20 years later. Am J Psychiatry 163:1001-1008.

9. Weissman MM, Wickramaratne $\mathrm{P}$, Nomura $\mathrm{Y}$, Warner $\mathrm{V}$, Verdeli $\mathrm{H}$, Pilowsky DJ, et al. (2005): Families at high and low risk for depression: A 3-generation study. Arch Gen Psychiatry 62:29-36.

10. Mannuzza S, Fyer AJ, Klein DF, Endicott J (1986): Schedule for Affective Disorders and Schizophrenia-Lifetime Version modified for the study of anxiety disorders (SADS-LA): Rationale and conceptual development. J Psychiatr Res 20:317-325. 
11. Kaufman J, Birmaher B, Brent D, Rao U, Flynn C, Moreci P, et al. (1997): Schedule for Affective Disorders and Schizophrenia for SchoolAge Children-Present and Lifetime Version (K-SADS-PL): Initial reliability and validity data. J Am Acad Child Adolesc Psychiatr 36: 980-988.

12. Fried PA, Watkinson B, Dillon RF, Dulberg CS (1987): Neonatal neurological status in a low-risk population after prenatal exposure to cigarettes, marijuana, and alcohol. J Dev Behav Pediatr 8:318-326.

13. Hamilton M (1967): Development of a rating scale for primary depressive illness. Br J Soc Clin Psychol 6:278-296.

14. Poznanski E, Mokros HB, Grossman J, Freeman LN (1985): Diagnostic criteria in childhood depression. Am J Psychiatry 142:1168-1173.

15. Fava M, Rosenbaum JF (1999): Anger attacks in patients with depression. J Clin Psychiatry 60(Suppl 15):21-24.

16. Perrin S, Last CG (1992): Do childhood anxiety measures measure anxiety? J Abnorm Child Psychol 20:567-578.

17. Patton JH, Stanford MS, Barratt ES (1995): Factor structure of the Barratt impulsiveness scale. J Clin Psychol 51:768-774.

18. Buss AH, Perry M (1992): The aggression questionnaire. J Pers Soc Psychol 63:452-459.

19. Gerevich J, Bacskai E, Czobor P (2007): The generalizability of the Buss-Perry Aggression Questionnaire. Int J Methods Psychiatr Res 16:124-136.

20. Han X, Jovicich J, Salat D, van der Kouwe A, Quinn B, Czanner S, et al. (2006): Reliability of MRI-derived measurements of human cerebral cortical thickness: The effects of field strength, scanner upgrade and manufacturer. Neuroimage 32:180-194.

21. Benjamini Y, Yekutieli D (2001): The control of the false discovery rate in multiple testing under dependency. Ann Stat 29:1165-1188.

22. Rucker DD, Preacher KJ, Tormala ZL, Petty RE (2011): Mediation analysis in social psychology: Current practices and new recommendations. Social and Personality Psychology Compass 5:359-371.

23. Koponen S, Taiminen T, Kurki T, Portin R, Isoniemi H, Himanen L, et al. (2006): MRI findings and Axis I and II psychiatric disorders after traumatic brain injury: A 30-year retrospective follow-up study. Psychiatry Res 146:263-270.

24. Kumar A, Bilker W, Jin Z, Udupa J (2000): Atrophy and high intensity lesions: Complementary neurobiological mechanisms in late-life major depression. Neuropsychopharmacology 22:264-274.

25. Rosenberg DR, Macmaster FP, Mirza Y, Smith JM, Easter PC, Banerjee SP, et al. (2005): Reduced anterior cingulate glutamate in pediatric major depression: A magnetic resonance spectroscopy study. Biol Psychiatry 58:700-704.

26. Rosso IM, Cintron CM, Steingard RJ, Renshaw PF, Young AD, Yurgelun-Todd DA (2005): Amygdala and hippocampus volumes in pediatric major depression. Biol Psychiatry 57:21-26.

27. Bora E, Fornito A, Yucel M, Pantelis C (2012): The effects of gender on grey matter abnormalities in major psychoses: A comparative voxelwise meta-analysis of schizophrenia and bipolar disorder. Psychol Med 42:295-307.

28. Eklund A, Nichols TE, Knutsson H (2016): Cluster failure: Why fMRI inferences for spatial extent have inflated false-positive rates. Proc Natl Acad Sci U S A 113:7900-7905.

29. Salmond $\mathrm{CH}$, Ashburner J, Vargha-Khadem F, Connelly A, Gadian DG, Friston KJ (2002): Distributional assumptions in voxel-based morphometry. Neuroimage 17:1027-1030.

30. Grieve SM, Korgaonkar MS, Koslow SH, Gordon E, Williams LM (2013): Widespread reductions in gray matter volume in depression. Neuroimage Clin 3:332-339.

31. Papmeyer M, Giles S, Sussmann JE, Kielty S, Stewart T, Lawrie SM, et al. (2015): Cortical thickness in individuals at high familial risk of mood disorders as they develop major depressive disorder. Biol Psychiatry 78:58-66.

32. Tu PC, Chen LF, Hsieh JC, Bai YM, Li CT, Su TP (2012): Regional cortical thinning in patients with major depressive disorder: A surfacebased morphometry study. Psychiatry Res 202:206-213.

33. van Tol MJ, Li M, Metzger CD, Hailla N, Horn DI, Li W, et al. (2014): Local cortical thinning links to resting-state disconnectivity in major depressive disorder. Psychol Med 44:2053-2065.

34. Kempton MJ, Salvador Z, Munafo MR, Geddes JR, Simmons A, Frangou S, et al. (2011): Structural neuroimaging studies in major depressive disorder. Meta-analysis and comparison with bipolar disorder. Arch Gen Psychiatry 68:675-690.

35. Ajilore O, Narr K, Rosenthal J, Pham D, Hamilton L, Watari K, et al. (2010): Regional cortical gray matter thickness differences associated with type 2 diabetes and major depression. Psychiatry Res 184:63-70.

36. Lebedev AV, Beyer MK, Fritze F, Westman E, Ballard C, Aarsland D (2014): Cortical changes associated with depression and antidepressant use in Alzheimer and Lewy body dementia: An MRI surface-based morphometric study. Am J Geriatr Psychiatry 22:4.e1-13.e1.

37. Weissman MM, Brown AS, Talati A (2011): Translational epidemiology in psychiatry: Linking population to clinical and basic sciences. Arch Gen Psychiatry 68:600-608.

38. Fava M, Rosenbaum JF, McCarthy M, Pava J, Steingard R, Bless E (1991): Anger attacks in depressed outpatients and their response to fluoxetine. Psychopharmacol Bull 27:275-279.

39. Papmeyer M, Sussmann JE, Stewart T, Giles S, Centola JG, Zannias V, et al. (2016): Prospective longitudinal study of subcortical brain volumes in individuals at high familial risk of mood disorders with or without subsequent onset of depression. Psychiatry Res 248:119-125.

40. Fava M, Anderson K, Rosenbaum JF (1990): "Anger attacks": Possible variants of panic and major depressive disorders. Am J Psychiatry 147:867-870.

41. Yang Y, Joshi SH, Jahanshad N, Thompson PM, Baker LA (2017): Neural correlates of proactive and reactive aggression in adolescent twins. Aggress Behav 43:230-240. 